\title{
AN APPLICATION OF A FIXED-POINT THEOREM TO NEUMANN PROBLEMS ON THE SIERPINSKI FRACTAL
}

\author{
BRIGITTE E. BRECKNER* AND CSABA VARGA** \\ Dedicated to prof. dr. Ioan A. Rus on the occasion of his $80^{\text {th }}$ birthday. \\ *Babeş-Bolyai University, Faculty of Mathematics and Computer Science \\ Kogălniceanu str. 1, 400084 Cluj-Napoca, Romania \\ E-mail: brigitte@math.ubbcluj.ro \\ ** Babeş-Bolyai University, Faculty of Mathematics and Computer Science \\ Kogălniceanu str. 1, 400084 Cluj-Napoca, Romania \\ E-mail: varga_gy_csaba@yahoo.com
}

\begin{abstract}
We prepare first the background for the study of Neumann problems on the Sierpinski fractal in the $N$-dimensional Euclidean space. Hereafter we apply the Leray-Schauder continuation principle to prove the existence of at least one solution of certain Neumann problems on this fractal. Key Words and Phrases: Sierpinski gasket, harmonic extension procedure, Leray-Schauder continuation principle, Neumann problem on the Sierpinski gasket.
\end{abstract}

2010 Mathematics Subject Classification: 28A80, 35J05, 35J25, 35J60, 46E20, 47H10, 49J52.

Acknowledgements. Csaba Varga has been partially supported both by a grant of the Romanian National Authority for Scientific Research (CNCS-UEFISCDI), grant no. PN-II-ID-PCE-2011-3-0241, and by a grant of the Hungarian Research Fund (OTKA), grant no. K 115926.

\section{REFERENCES}

[1] H. Bauer, Measure and Integration Theory, de Gruyter Studies in Mathematics, vol. 26, Walter de Gruyter \& Co., Berlin, 2001.

[2] B.E. Breckner, Real-valued functions of finite energy on the Sierpinski gasket, Mathematica, 78(2013), no. 2, 142-158.

[3] B.E. Breckner, R. Chill, The Laplace operator on the Sierpinski gasket with Robin boundary conditions, Nonlinear Anal. Real World Appl., 38(2017), 245-260.

[4] B.E. Breckner, Cs. Varga, Elliptic problems on the Sierpinski gasket, Topics in Mathematical Analysis and Applications (Eds. Th. M. Rassias, L. Toth), Springer Optimization and its Applications 94, Springer, 2014, 119-173.

[5] K.J. Falconer, J. Hu, Non-linear elliptical equations on the Sierpinski gasket, J. Math. Anal. Appl., 240(1999), 552-573.

[6] J. Kigami, A harmonic calculus on the Sierpinski spaces, Japan J. Appl. Math., 6(1989), 259290.

[7] J. Kigami, Harmonic calculus on p.c.f. self-similar sets, Trans. Amer. Math. Soc., 335(1993), 721-755. 
[8] J. Kigami, Analysis on Fractals, Cambridge University Press, Cambridge, UK, 2001.

[9] D. O'Regan, R. Precup, Theorems of Leray-Schauder Type and Applications, Gordon and Breach Science Publ., Amsterdam, 2001.

[10] R.S. Strichartz, Differential Equations on Fractals. A Tutorial, Princeton Univ. Press, Princeton, NJ, 2006.

[11] E. Zeidler, Nonlinear Functional Analysis and its Applications, vol. I, Springer-Verlag, 1986.

Received: March 24, 2016; Accepted: May 25, 2016. 
